\title{
Efficacy of purified nucleotide supplements on the growth performance and immunity of hybrid striped bass Morone chrysops $x$ Morone saxatilis
}

\begin{abstract}
Fishmeal is being increasingly replaced in aquatic animal diets with alternative plant protein feedstuffs such as soybean meal which have lower concentrations of nucleotides; therefore, supplemental sources of exogenous nucleotides in diets could become increasingly important. A 9-week feeding trial was conducted with triplicate groups of juvenile hybrid striped bass (average initial body weight \pm standard deviation, $5.6 \pm 0.1 \mathrm{~g}$ ) to determine the effects of supplementing single purified nucleotides on the growth performance and immune parameters. The basal diet, which utilized menhaden fishmeal $(25 \%)$ and soybean meal $(75 \%)$ as protein sources, contained $44 \%$ protein, $10 \%$ lipid and an estimated digestible energy level of $3.5 \mathrm{kcal}$ g- 1 . Single additions of 5'- adenosine monophosphate (AMP), 5'- uridine monophosphate (UMP), 5'- cytidine monophosphate (CMP), 5'- guanosine monophosphate (GMP), and 5'- inosine monophosphate (IMP) disodium salts (Chem-Impex International, Wood Dale, Illinois, USA) were evaluated with each nucleotide added to the basal diet at $0.5 \%$ of dry weight at the expense of cellulose. A positive control diet in this trial was a diet containing 5'- AMP from Sigma-Aldrich also supplemented at $0.5 \%$ by weight. Results showed significantly $(\mathrm{P}<0.05)$ improved weight gain between fish fed AMP-supplemented diets and the basal diet. No statistical significance $(\mathrm{P}>0.05)$ was detected in whole-body proximate composition and protein retention of fish fed any of the dietary treatments. The respiratory burst of whole blood phagocytes also was significantly $(\mathrm{P}<0.05)$ higher in fish fed the AMP Sigma diet compared to the other dietary treatments. Dietary IMP and AMP both significantly $(\mathrm{P}<0.05)$ enhanced the capacity of isolated phagocytes to generate extracellular superoxide anion compared to all other dietary treatments. No significant differences were seen in other innate immune parameters such as plasma lysozyme, total plasma protein, and total immunoglobulin. The ability of isolated B lymphocytes to proliferate prompted by the presence of lipopolysaccharides was significantly $(\mathrm{P}<0.05)$ different among dietary treatments with the highest simulation index observed in fish fed the diets containing AMP Sigma and UMP; however, it was not significantly different from that of fish fed the basal diet. Based on all the measured responses, it is concluded that AMP at $0.5 \%$ of diet had the most positive influence on growth performance and innate immunostimulation of hybrid striped bass.
\end{abstract}

Keyword: AMP; Hybrid striped bass; IMP; Immunomodulation; Nucleotides 